Apidologie, 1971, 2 (3), 271-282.

\title{
ÜBER DEN « ORTSFESTEN » FREIEN FLUG VON BIENEN IN EINEM SAUGKANAL
}

\author{
Sur le vol « stationnaire » libre de l'abeille en soufflerie
}

Werner NACHTIGALL, Reinhardt WIDMANN und Maximilian RENNER

Zoologische Institute der Universität des Saarlandes, Saarbrïcken, und der Universitäi München.

\section{SUMMARY}

ON THE FREE (C STATIONARY ) FLIGHT OF THE BEE IN A WIND TUNNEL

Bees were trained to fly from their hive to a feeding dish via a suction type of wind tunnel and to return to the hive via another route outside the wind tunnel. While going through the tunnel the insect flew against the wind, the velocity of which could be regulated so that it was exactly equal to the flight velocity of the bee. As a result, the bee flew ' in place'. Some bees maintained this flight condition as long as 20 seconds. This behavior and the experimental apparatus make possible high-frequency kinematic analysis of freely flying bees. The maximal velocity of the bees in the wind tunnel did not exceed $5.5 \mathrm{~m} / \mathrm{s}$. Rhythmic swings of the animal in a plane perpendicular to the air stream were observed. Visual stimuli consisting of green and yellow stripes moved in a direction opposite to the flight direction thus far have not influenced the flight behavior of the animals. The angle of inclination of the bees' body and the variation in this angle decreased significantly with increasing velocity of flight. Parameters of the flight behavior were discussed.

\section{ZUSAMMENFASSUNG}

Bienen wurden darauf dressiert, in einem Saugkanal gegen die Luftströmung zu einem Futterschälchen zu fliegen und auf einem anderen Wege, außerhalb des Kanals, zum Stock zurückzukehren. Die Strömungsgeschwindigkeit kann so reguliert werden, daß die Fluggeschwindigkeit der Biene im Kanal entgegengesetzt gleich der Windgeschwindigkeit ist, so daß das Tier' am Ort' fliegt. Einzelne Individuen halten diesen Flugzustand bis zu $20 \mathrm{~s}$ durch, so daß die hochfrequenzkinematische Analyse freifliegender Bienen mit der beschriebenen Versuchseinrichtung möglich ist. Die Maximalgeschwindigkeit der Bienen im Saugkanal über- 
schreitet nicht $5,5 \mathrm{~m} / \mathrm{s}$. Rhythmische Seitschwankungen treten auf. Gegenbewegte optische Marken haben bisher keinen Einfluß auf das Flugverhalten gezeigt. Der Rumpfanstellwinkel der Bienen und die Streuung des Rumpfanstellwinkels sinken statistich gesichert mit steigender Fluggeschwindigkeit. Die Parameter des Flugverhaltens werden diskutiert.

\section{EINLEITUNG}

Als Standardverfahren für flugbiophysikalische Untersuchungen von Insekten hat sich folgende Methode herausgebildet : Das Insekt wird an einer Mehrkomponentenwaage fixiert und von einem Windkanal laminar angeströmt. Sobald die Anströmungsgeschwindigkeit gleich der Fluggeschwindigkeit ist, und sobald die Kraftkomponenten Auftrieb-Gewicht und VortriebWiderstand jeweils entgegengesetzt gleich groß sind, herrschen quasinatürliche Verhältnisse. Man kann dann erwarten, daß die Flügel in gleicher Weise schlagen, als wenn das Insekt mit der eingestellten Geschwindigkeit horizontal geradlinig durch ruhende Luft flöge. Mit geeigneten, auswertbaren Registrierverfahren, etwa stereographischen Hochfrequenz-Filmaufnahmen, kann man dann die Kinematik der Flügelschwingung aufnehmen.

Dieses Prinzip wurde u.W. von HoLlick (1940) an der Fliege Muscina stabulans das erste Mal systematisch angewendet. Im folgenden wurde es verbessert und weiterentwickelt, insbesondere von JENsEN (1956) bei der Heuschrecke Schistocerca gregaria (zweidimensionale Filmaufnahmen), von Nachtigall (1966) bei der Glanzfliege Phormia regina (sterographische Dreitafelprojektion), von Vogel (1966) bei der Fruchtfliege Drosophila virilis (zweidimensionale Photos) und von Zarnack (1970) bei der Wanderheuschrecke Locusta migratoria (Stereo-Meßbildpaare). Die Bienen haben insbesondere Neuhaus und Wohlgemuth (1960), Wohlgemuth (1962) und Herbst und Freund (1962) mit Windkanal-Methoden untersucht. Der Hauptnachteil dieses Verfahrens ist die Aufhängung des Tieres. Thorakale Aufhängungen müssen Massenbeschleunigungen hervorrufen, die im freien Flug nicht auftreten, und die möglicherweise die Flügelkinematik, sicher jedenfalls die Flugenergetik verändern. Allein mit Aufhängungen über ein sehr zartes Haltegestänge an der äußersten Abdomenspitze (Nachtigall (1966)) kann ein (C quasinatürlicher ) Flugzustand eingestellt werden.

Solche Aufhängungen sind aber methodisch sehr heikel. Es wäre deshalb von großem Vorteil, wenn es gelänge, Tiere im vollständig freien Flug zu untersuchen. Für die Analyse der Flügelkinematik, für Fragen der Treibstoffverwertung (Energetik) und für die Untersuchung der Flugsteuerung und der Lageorientierung ist eine völlig störungsfreie Simulation des freien Flugzustandes sehr bedeutsam. Zur Beobachtung muß das Tier relativ zu seiner Umgebung « ortsfest » fliegen. Das läßt sich technisch dadurch erreichen, daß man es aus einem Saugkanal herausfliegen läßt, in den man so rasch Luft einsaugt, daß die Sauggeschwindigkeit entgegengesetzt gleich der Flug- 
geschwindigkeit ist. Nicht-dressurfähige Insekten, wie zum Beispiel Fliegen, sind für solche Versuche ungeeignet. Bienen dagegen können es lernen, von einer Abflugrampe in der langgestreckten Meßkammer eines Saugkanals aus zu einer Futterquelle wegzufliegen. Da bei den Dressurexperimenten einige methodisch interessante Ergebnisse aufgetreten sind, die insbesondere den Bienenpraktiker interessieren dürften, sei hier etwas näher darauf eingegangen.

\section{METHODE}

Die Versuche wurden in dem $6 \mathrm{~m}^{2}$ großen Bienenraum des Zoologischen Instituts der Universität München ausgeführt [Renner, M. $(1955,1957)]$. Die Wände des Raumes waren auf weißem Grund mit gelben und grünen Orientierungsmalen versehen. Der Raum wurde künstlich beleuchtet, belüftet und auf $25^{\circ} \mathrm{C}$ temperaturkonstant gehalten. Die relative Luftfeuchtigkeit wurde durch Verdunstungsflächen auf etwa $50 \%$ eingestellt. Die Leuchtstoffröhren-Deckenbeleuchtung wurde automatisch für eine Tageslänge von 14. Stunden (5 bis 19 Uhr) mit Dämmerungsphasen von je 20 Minuten geschaltet. Während der Tagesperiode erzeugte sie in $1 \mathrm{~m}$ Höhe über dem Boden eine mittlere Raumhelligkeit von 500 Lux. Die abendliche Dämmerungsphase ermöglichte den Bienen die Rückkehr zum Stock. Gefüttert wurde mit zweimolarem Zuckerwasser. Diese Konzentration wurde von den Bienen am besten angeflogen. Die für die Brut nötige eiweißhaltige Nahrung bestand aus handelsüblichen Höselpollen. Zur Förderung der Kotabgabe und um eine Erkrankung an Nosema zu verhindern, wurde regelmäßig süßer Sennesblättertee eingefüttert, dem pro $100 \mathrm{~g} 0,1 \mathrm{~g}$ Fumagillin zugesetzt war.

Der verwendete Windkanal ist in Abbildung 1 maßstabsgetreu aufgezeichnet. Eine kräftige, einstufige Achsialturbine, angetrieben von einem 2 PS-Drehstrommotor, saugt Luft aus der Umgebung durch eine aerodynamisch günstig geformte runde Einströmdüse und durch die $80 \mathrm{~cm}$ lange und $12 \times 12 \mathrm{~cm}$ im Querschnitt messende Beobachtungsstrecke. Windgeschwindigkeiten von max. $6 \mathrm{~m} / \mathrm{s}$ wurden damit erreicht. Reguliert wurde die Geschwindigkeit durch teilweises Schließen einer Schlitzblende am Ausströmende (Einschaltbild in Abb 1). Die Blendenstellung wurde mit einem Flügelradanemometer in Geschwindigkeitseinheiten geeicht.

Die Meßstrecke bestand aux Glas und konnte zum Reinigen leicht geöffnet werden. Durch die Düse und mittles vorgespanneter Perlonsiebe der feinsten Maschenweite wurde die Raumturbulenz nahezu ausgeschaltet. Rauchfadenversuche zeigten bis zu mittleren Gesehwindigkeiten parallele, unverwirbelte Stromfäden in der Meßstrecke. Der Kanal konnte um $\pm 30^{\circ}$ geneigt werden, wurde aber bei den hier beschriebenen Versuchen stets in horizontaler Stellung benutzt. Vom Stock zog ein Gummischlauch zentral in die Meßstrecke und endete an einer Startplattform. Die Bienen wurden darauf dressiert, von der Startplattform gegen den Wind zu fliegen, auf der Innenseite des innersten Siebes zu landen, nach oben zu einem seitlich an der Düse angebrachten Austrittsloch zu kriechen und von dort zu einem Futterschälchen zu fliegen. Das Schälchen stand in ca. $1 \mathrm{~m}$ Abstand von der Düse in Höhe der Kanalmittelachse und wurde von 2 Niedervoltlampen sehr hell angestrahlt, so daß es als heller Fleck vom Kanal aus sichtbar war. Als besonders günstig erwies sich eine Blaufärbung des Schälchens.

\section{DAS VERHALTEN DER BIENEN UNTER VERSUCHSBEDINGUNGEN ENTWICKLUNG DER VERSUCHSEINRICHTUNG}

Die Versuchseinrichtung wurde schrittweise im steten Test mit den Verhaltensleistungen der Bienen entwickelt. Anfangs wurden Einzeltiere verwendet; sie sind jedoch kaum zum Abflug zu veranlassen. Deshalb wurde schließlich ein Bienenvolk verwendet. Die Tiere waren vorher schon an die Bedingungen des Bienenraums und an die künstliche Fütterung gewöhnt worden. 


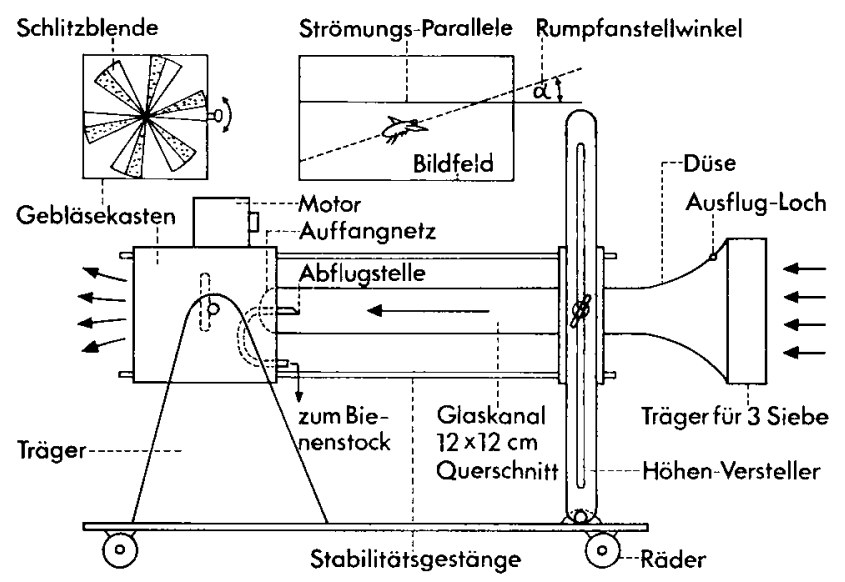

Aвв. 1. - Konstruktionsskizze des verwendeten Saugkanals. Einschaltbilder : Blende zur Geschwindigkeitsregelung an der Ausströmöffnnug; Definition des Rumpfanstellwinkels.

FIG. 1. - Schéma de la soufflerie utilisée. En haut et à gauche : diaphragme pour le réglage de la vitesse du courant d'air. En haut et au centre : définition de l'angle d'incidence du corps de l'abeille.

Schlitzblende : diaphragme.

Strömungs-Parallele : parallèle au courant.

Rumpfanstellwinkel : angle 'd'incidence du corps.

Bildfeld : champ de l'image.

Gebläsekasten : boîte du ventilateur.

Motor : moteur.

Auffangnetz : grillage.

Abflugstelle : point d'envol.

Düse : tuyère.

Ausflug-Loch : trou de sortie.

Träger : support.

zum Bienenstock : vers la ruche.

Glaskanal $12 \times 12$ Querschnitt : canal vitré $12 \times 12 \mathrm{crn}$ de section.

Träger für 3 Siebe : support pour 3 cribles métalliques.

Höhen-Versteller : réglage de la hauteur.

Stabilitätsgestänge : bâti de stabilisation.

Räder : roues.

Stockausgang und Abflugstelle waren mit einem Gummischlauch von $70 \mathrm{~cm}$ Länge und $2 \mathrm{~cm}$ Innendurchmesser verbunden. Ohne Dressur fanden sich die Bienen in dem Gummirohr nicht zurecht. Sie wurden deshalb mit Stutzen von lo, 20 und $30 \mathrm{~cm}$ Länge an die neue Art des Stockausgangs gewöhnt das Futter befand sich jeweils direkt am Schlauchende und war mit einer Duft- und Lichtquelle kombiniert. Von der Startplattform im Kanal sollten die Tiere gegen den Wind auf Nahrung in einem stromlinienförmig verkleideten Futtergefäß dressiert werden, das zunächst ganz nahe, später immer weiter entfernt, schließlich außerhalb des Kanals stehen sollte. Die Bienen flogen das Gefäß jedoch nicht an. Deshalb wurde das Gefäß außerhalb des Kanals angebracht. Das seitliche Ausflugsloch wurde hell angestrahlt. Die Tiere fanden aber nicht ins Freie. Zufällig wurde einmal statt des Turbulenzsiebs eine licht-und strömungsundurchlässige Ringblende angebracht. Jetzt landeten die Tiere am Grund der Einströmdüse und akzeptierten das kleine Ausflugsloch. Nach 3 Tagen wurde die Blende gegen das Turbulenzsieb ausgetauscht. Eine 
Niedervoltlampe projizierte einen kreisförmigen Lichtfleck ins Zentrum des Siebes; die dunkle Randzone entsprach der vorher verwendeten Ringblende. Die Blende wurde somit vorgetäuscht; die Bienen akzeptierten diese Situation. Nach weiteren 14 Tagen konnte die kreisförmige Beleuchtung des Siebes und die Anleuchtung des Ausflugslochs wegfallen. Die Bienen durchwanderten nun regelmäßig den Kreislauf Stock-Gummischlauch-Abflugrampe-freier Flug gegen den Wind (sehr langsames Vorankommen) zum Sieb (Einschränkungen s.u.) - aufwärts Laufen - Auskriechen aus dem Ausflugloch - Flug zur Futterquelle - Flug direkt zum Stock zurück.

Für Versuche dieser Art ist es ausschlaggebend wichtig, ob Bienen lernen können, auf getrennten Wegen aus dem Stock zu gelangen (hier : durch Schlauch und Windkanal) und in den Stock zurückzukehren (hier : durch Eingangsöfnung am Stock selbst), da es - bei dieser komplizierten Versuchsanordnung verständlicherweise - nicht gelang, die Bienen dazu zu bringen, « mit Wind » auf umgekehrtem Wege durch den Kanal in den Stock zurückzukehren. Sie flogen nach dem Saugen am Schälchen direkt an den Stock und suchten an der Aussenwand nach einer Öffnung. Es wurde deshalb ein Loch von $15 \mathrm{~mm}$ Durchmesser in die Stockwand gebohrt und innen mit einem etwas größeren Plastikplättchen verschlossen, das an der Oberseite drehbar aufgehängt war. Dieses Klapptürchen konnte von den rückkehrenden Bienen mit einigen Mühen aufgedrückt werden, infolge der Innenlagerung jedoch nicht von den im Stock befindlichen Tieren. Es wurde bald von allen Sammlerinnen als Stockeingang akzeptiert.

Eine große Schwierigkeit lag darin, daß viele Bienen im Wind nur ungern von der Startplattform wegfliegen und lieber auf Umwegen an der Kanalwand entlang krabbeln und sich dann zu Fuß zum Ausflugloch begeben. Folgende Verfahren, die die Bienen zum Abfliegen veranlassen sollten, wurden getestet :

1. Start in ruhender Luft; schlagartiges Einschalten der Turbine nach dem Start. Die Bienen wurden regelmäßig von der Einschaltbö gegen die Kanalwand geworfen und ins Auffangnetz zurückgetrieben. Gleitendes Anlaufen über photomechanische Selbststeuerung könnte Abhilfe bringen, konnte jedoch nicht getestet werden.

2. Umgeben der Startplattform mit öligen, wässrigen, klebrigen, wächsernen und nadelpolsterartigen Überzügen, die nicht überklettert werden können. Bei Wind laufen viele Tiere rasch und ungerichtet weg, verfangen sich an dem Hindernis und fallen zu Boden.

3. Ein in bestimmter Weise geformter Ausflugtrichter, in dem die Windgeschwindigkeit vom Staupunkt aus graduell zunimmt. Die Tiere laufen jedoch bis zum Ende des Trichters.

4. Schlagartiges Wegreißen der Startplattform über einen kräftigen Elektromagneten in ca. 3 ms. Die Tiere fallen daraufhin frei durch und könnten dabei reflektorisch starten. Die Fallstrecke reichte aber nicht aus. 
5. Vergrößern der Fallstrecke durch einen größeren Kanalquerschnitt $(30 \times 30 \mathrm{~cm})$. Trotz Start und Flügelbewegung wurden die Tiere regelmäßig rückwärts oder gegen die Seitenwände abgetrieben.

Somit blieb nur der Weg, diejenigen Tiere abzuwarten und zu beobachten, die zufällig spontan und gut starteten und weiterhin gut flogen. Es waren etwa $2 \%$ aller Tiere; die übrigen liefen meist die Glaswände entlang zum Ausflugloch oder flogen schlecht (Abb. 2).

a)
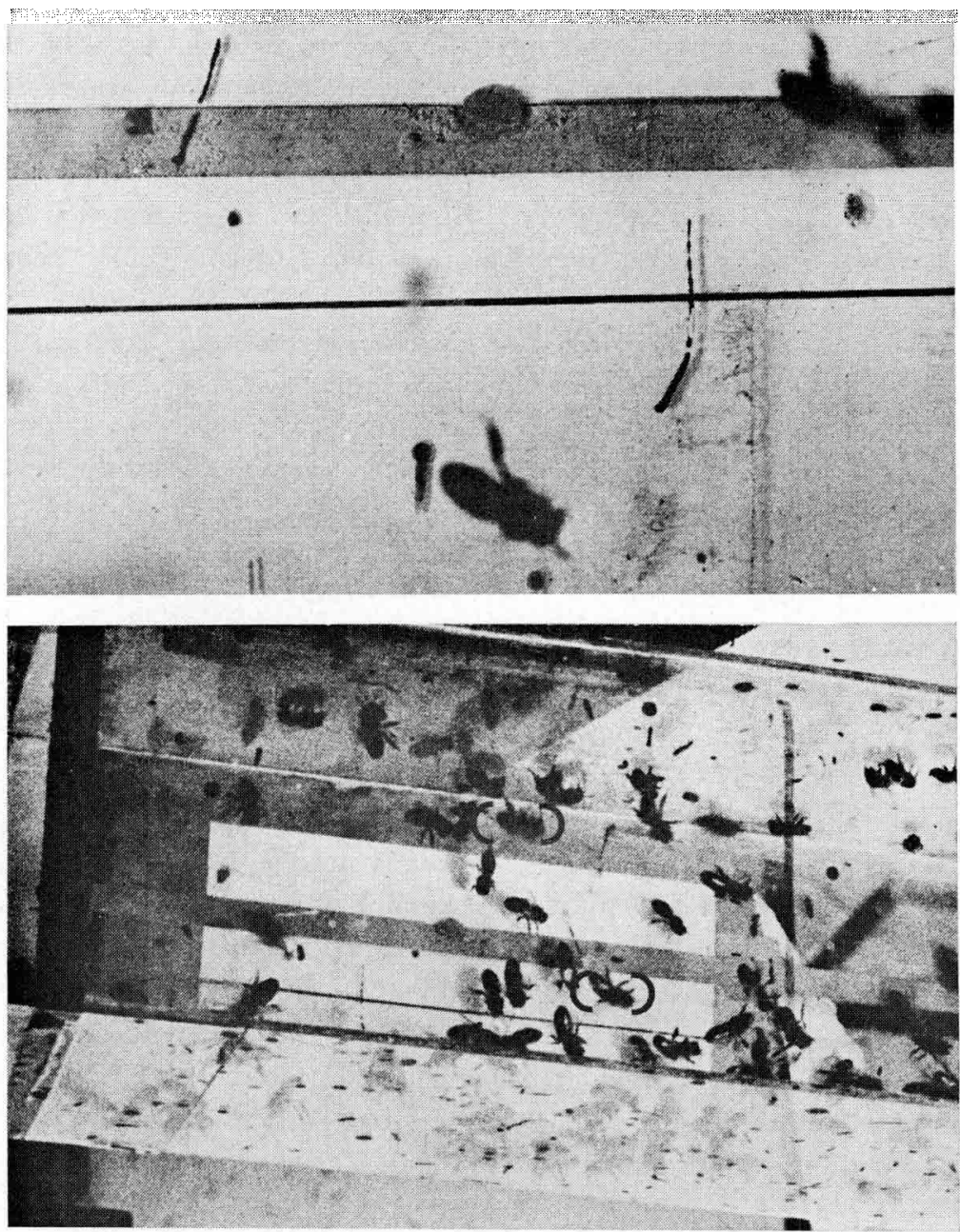

Aвв. 2. - a) Durchschnittliche Situation in der Meßstrecke des Saugkanals : die meisten Bienen laufen, nur 2 Bienen (Halbkreise) fliegen hier gut und stetig. b) Einzeln fliegende Biene bei $v=5 \mathrm{~m} / \mathrm{s}$. Der dünne Strich entspricht der Windrichtung. Der Wind kommt von links.

Fig. 2. - a) Situation moyenne dans la veine du canal d'aspiration : la plupart des abeilles marchent, seultment 2 abeilles (signalées par des demi-cercles) volent correctement et régulièrement. b) Quelques abeilles volant à $v=5 \mathrm{~m} / \mathrm{s}$. Le trait mince continu indique la direction du vent qui vient de la gauche. 


\section{DAS VERHALTEN DER BIENEN BEIM FREIEN FLUG IM SAUGKANAL}

Tiere, die gut gestartet waren, flogen mit geschwindigkeitsabhängigen Rumpfanstellwinkeln (s.u.) und in der typischen Flughaltung für langsame bis mittlere Geschwindigkeiten : Vorder- und Mittelbeine mehrminder angezogen, Hinterbeine schräg nach hinten - unten herabhängend. Sie bewegten sich frontal gegen den Wind. Durch Nachregeln der Windgeschwindigkeit von Hand konnten sie leicht vor- und zurückgetrieben werden. Sie können auch - spontan oder infolge Regelung - mit einigen wenigen Zentimetern Schwankungsbreite ( am Ort ) fliegen. Solche Flugzustände waren jedoch nicht häufig und hielten meist nur $5-10$, höchstens 20 Sekunden an. Bei einer Fluggeschwindigkeit von $5 \mathrm{~m} / \mathrm{s}$ entspricht eine Flugdauer von 20 Sekunden einer zurückgelegten Strecke von $100 \mathrm{~m}$. Bereits 1 Sekunde eines derartigen freien Stationärflugs reicht zur kinematischen Analyse der Flügelschwingungen vollständig aus. Unter diesem Gesichtspunkt hat sich das Verfahren als erfolgreich erwiesen. Für energetische Untersuchungen müßte die genaue Fluggeschwindigkeit, beziehungsweise ihre zeitliche Änderung, präzise bekannt sein. Freifliegende Bienen führen nun aber im Kanal sehr heftige, offensichtlich spontane Richtungsschwankungen aus, die sie oft in die Nähe der Kanalwände treiben und nicht selten anstoßen lassen. Auch im größeren $30 \times 30 \mathrm{~cm}-\mathrm{Kanal}$ war das Verhalten nicht anders. Feste optische Marken am Außenrand der gläsernen Meßstrecke [vergl. Heran und Lindauer (1963)], die verschieden hell beleuchtet werden konnten, verringerten diese Seitschwankungen ebenfalls nicht. Die Flugzustände sind also nicht definiert, so daß die Methode für Analysen des Energiehaushalts nicht geeignet sein dürfte.

Es ist nicht bekannt, ob die Bienen die rhythmischen Schwankungen aktiv ausführen, ob diese Bewegungen durch eventuelle Restturbulenz im Kanal induziert werden, oder ob die nur einige wenige Zentimeter betragenden Schwankungen auch beim freien Flug in ruhender Luft als Normalverhalten auftreten, möglicherweise aber infolge der meist großen Beobachtungsentfernungen und Fluggeschwindigkeiten übersehen werden : die Seitschwankungs-Geschwindigkeiten sind meist klein gegen die Fluggeschwindigkeit. Fluggeschwindigkeiten - hier identisch mit Gegenwindgeschwindigkeiten von $0-5,5 \mathrm{~m} / \mathrm{s}(20 \mathrm{~km} / \mathrm{Std}$.) wurden im Kanal erreicht. Im freien Gelände sind Geschwindigkeiten um $8 \mathrm{~m} / \mathrm{s}$ (30 km/Std.) und etwas mehr sicher nachgewiesen. Der Grund dafür, warum die Bienen ihre physiologische Höchstgeschwindigkeit im Kanal nicht ausfliegen, ist nicht bekannt. Möglicherweise können die im Bienenraum gehaltenen Tiere infolge des Zwangs zum ständigen Schwirr- bis Langsamflug die höchsten Geschwindigkeiten nicht mehr entwickeln. Eine weitere Erklärungsmöglichkeit wird am Schluß der Mitteilung gegeben.

Die im Saugkanal auftretende Relativgeschwindigkeit Biene - Umgebung 
ist beim stationären Flug gleich Null. Zur Kompensation für den fehlenden bewegungsoptischen Reiz wurde ein Streifenband geboten $(80 \mathrm{~cm}$ lang, $12 \mathrm{~cm}$ breit, gelbe und grüne Streifen je $5 \mathrm{~cm}$ tief), das unmittelbar unter der gläsernen Bodenplatte der Meßstrecke gegen die Flugrichtung lief und in verschiedenen Ausschnitten und verschieden hell beleuchtet werden konnte. Seine Relativgeschwindigkeit konnte der Windgeschwindigkeit angeglichen werden. Ein gesicherter Einfluß bezüglich einer Erhöhung der Fluggeschwindigkeit und einer Stabilisierung des Flugverhaltens konnte mit der verwendeten Apparatur bisher nicht nachgewiesen werden.

\section{RUMPFANSTELLWINKEL UND FLUGGESCHWINDIGKEIT}

Als ein Beispiel für die Meßmöglichkeiten, die ein solches Kanalsystem erlaubt, sei der Rumpfanstellwinkel als Funktion der Fluggeschwindigkeit betrachtet. Dieser Winkel sei hier definiert als Winkel zwischen der Tangente an die obere Körperkontur der Biene und der Strömungsrichtung (s. Abb. 1, Einschaltbild). Bei größerer Fluggeschwindigkeit muß mehr Vortrieb relativ zum Auftrieb erzeugt werden. Setzt man hypothetisch voraus, daß die Flügelkinematik und die mittlere Schlagbahnneigung zur Tierlängsachse konstant bleibt, so müssen die Rumpfanstellwinkel mit steigender Fluggeschwindigkeit abnehmen, das heißt die Schlagbahnneigung zur Horizontalen muß steiler und der Rumpfwiderstand kleiner werden.

Zusammen mit einer strömungsparallelen Hintergrundmarkierung wurde ein mittlerer Ausschnitt der exakt horizontal eingestellten Meßstrecke auf $16 \mathrm{~mm}$ - Film gefilmt. Die optische Kameraachse stand horizontal und senkrecht zur Kanallängsachse. Bei großer Blende wurde mit folgender, für die Analyse optimaler Einstellung gefilmt : 8 Bildern pro Sekunde; Einzelbildbelichtungszeit mittels Sektorenblende auf $1 / 32$ s eingestell. Infolge der offenen Blende wurden nur zentral fliegende Bienen scharf abgebildet. Wegen der relativ langen Einzelbildbelichtungszeit wurden weiter nur diejenigen Tiere scharf erfaßt, die stationär flogen. Stationäre Flugzustände konnten weiter noch daran erkannt werden, daß das vorhergehende und das nachfolgende Filmbild die Biene in gleicher Raumlage, zumindesten in gleicher Höhenlage zeigen mußte. Schrägstellungen zur Strömung durch Gieren um die Hochachse (Schiebeflug), die Anstellwinkeländerungen vortäuschen würden, konnten dadurch bemerkt werden, daß die beiden schräg herabhängenden Hinterbeine in solchen Fällen nicht deckungsgleich sind. Bilder von instationären und nicht strömungsorientierten Flugzuständen wurden nicht verwertet. Bei Geschwindigkeiten von $0,2,3,4$ und $5 \mathrm{~m} / \mathrm{s}$ wurden jeweils loo Einzelbilder ausgewertet, die die genannten Kriterien präzise erfüllten. Die Ergebnisse sind in der Tabelle $I$ und in der Abbildung 3 zusammengefaßt. 


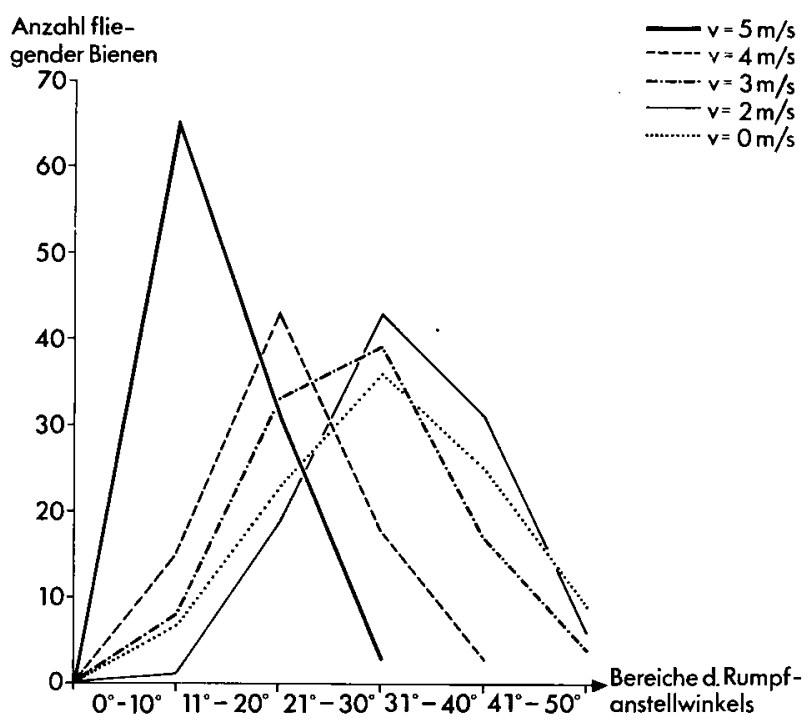

Авв. 3. - Häufigkeitsverteilung der gruppierten Rumpfanstellwinkel bei verschiedenen Fluggeschwindigkeiten. Die Abszissen-Intervalle gelten jeweils für die rechts stehenden Markierungen. Mit steigender Geschwindigkeit nehmen die Mittelwerte und die Varianzen der Verteilungen ab.

FIG. 3. - Répartition des fréquences des angles d'incidence du corps groupés pour différentes vitesses de vol. Les intervalles des abscisses sont valables pour les différentes conventions de traits figurées à droite. Lorsque la vitesse augmente les valeurs moyennes et les variances de la répartition diminuent.

Anzah fliegender Bienen : nombre d'abeilles qui volent.

Bereiche des Rumpfanstellwinkels : valeurs de l'angle d'incidence du corps.

Es hat sich gezeigt, daß der Rumpfanstellwinkel mit zunehmender Geschwindigkeit gesichert kleiner wird. Weiter hat sich ergeben, daß die Streuung des Rumpfanstellwinkels mit zunehmender Fluggeschwindigkeit gesichert abnimmt. Die Ursachen für das letztere Verhalten dürften darin liegen, daß die Tiere den für eine bestimmte Fluggeschwindigkeit typischen (energetisch optimalen ?) Rumpfanstellwinkel aus energetischen Gründen um so schärfer einhalten, je größer die Fluggeschwindigkeit ist. Ist dieser Winkel ein Optimum, so muß jede Abweichung um so energiezehrender sein, je schneller das Tier fliegt : Ausdrehmomente und Widerstandskräfte wachsen mit dem Quadrat der Fluggeschwindigkeit.

Erwähnenswert ist ferner, daß die Bienen bereits bei $5 \mathrm{~m} / \mathrm{s}$ sehr flach fliegen; der Rumpfanstellwinkel beträgt dabei im Durchschnitt nur etwa 90, in nicht wenigen Einzelfällen bereits nahe $0^{\circ}$. Möglicherweise sind Geschwindigkeiten etwas über $5 \mathrm{~m} / \mathrm{s}$ die höchsten Werte, die eine Biene durch Verringerung des Rumpfanstellwinkels einstellen kann, der wiederum in direkter mechanischer Beziehung zur Flügelamplitude stehen dürfte. Geschwindigkeitsmessungen im Freien ergaben Höchstwerte von 8-9 m/s [Heran (1959)]. $11 \mathrm{~m} / \mathrm{s}$ werden als mögliche Höchstgeschwindigkeit betrachtet. Weitere Steuerkomponenten — zusätzliche Veränderung der Schlagebene, der Phasenlage 
TAв. 1. - Mittelwerte ( $x$ ) und Streuungen ( $s$ ) der gemessenen Rumpfanstellwinkel bei verschiedenen Stromungsgeschwindigkeiten $(v) . n=Z a h l$ der Messengun pro Geschwindigkeitsstufe $=100$. Zur Sicherung der Unterschiede der Mittelwerte - bei verschiedenen Strömungsgeschwindigkeiten $v$ wurden $t$-Teste nach STUDENT ausgeführt. Unterschiede in den durchschnittlichen Anstellwinkeln für die Geschwindigkeitsstufen $(\mathrm{m} / \mathrm{s}) \mathrm{O}$ und 2, sowie 2 und 3 lassen sich nicht sichern; die Sicherung der übrigen Unterschiede ist hoch signifikant $(p=1 \%)$. Zur Sicherung der Unterschiede der Varianzen $s^{2}$ bei verschiedenen Strömungsgeschwindigkeiten wurden F-Teste nach Fisher durchgeführt. Unterschiede in den Varianzen für die Geschwindigkeitsstufen $(\mathrm{m} / \mathrm{s}) 3$ und 4 lassen sich nicht sichern; die übrigen Unterschiede sind signifikant $(p=5 \%)$.

TABL. 1. - Valeurs moyennes $(\bar{x})$ et dispersions $(s)$ des angles d'incidence du corps, mesurés pour différentes vitesses du courant d'air $(v) . n=$ nombre des mesures pour chacune des vitesses $=100$. Le test $t$ de STUDENT a été utilisé pour contrôler les différences des valeurs moyennes de $\bar{x}$ selon les vitesses du courant d'air $v$. Les différences de l'angle moyen pour les vitesses 0 et 2 , ainsi que 2 et 3 ne sont pas statistiquement significatives. Par contre les autres différences sont hautement significatives $(p=1 \%)$. Pour contrôler les différences entre les variances $s^{2}$ à diverses vitesses, on a utilisé le test $F$ de Fisher. Les différences de variances pour les vitesses 3 et $4(\mathrm{~m} / \mathrm{s})$ ne sont pas statistiquement significatives. Les autres différences sont significatives $(p=5 \%)$.

\begin{tabular}{c|c|c|c|c|c}
\hline \hline $\begin{array}{c}v[\mathrm{~m} / \mathrm{s}] \\
\begin{array}{c}\text { Statistische } \\
\text { Kenngrößen }\end{array}\end{array}$ & 0 & 2 & 3 & 4 & 5 \\
\hline $\bar{x}^{\circ}$ & 32,56 & 24,96 & 23,60 & 12,69 & 9,35 \\
\hline$s^{\circ}$ & 10,54 & 13,40 & 7,60 & 8,05 & 5,15 \\
\hline
\end{tabular}

zwischen Biege- und Drehschwingung der Flügelschläge etc. - könnten vielleicht gerade von der mit den Kanalexperimenten erzielbaren höchsten Geschwindigkeitsstufe $(5 \mathrm{~m} / \mathrm{s})$ ab dazukommen. Sie könnten die Geschwindigkeit auch dann noch höher treiben, wenn der Rumpfanstellwinkel gleich Null geworden ist. Solche Verstellungen kommen durch tonische Kontraktionen bestimmter direkter Stellmuskeln an der Flügelbasis zustande (Pringle 1961). Rückläufig argumentierend könnte man also nicht ausschließen, daß unter den Lebensbedingungen des Bienenraums diejenigen direkten Flugmuskeln geschädigt werden oder zumindesten nicht optimal arbeiten, die bei hohen Fluggeschwindigkeiten besonders aktiv sind.

Eine weitere Erklärungsmöglichkeit, warum die Bienen im Kanal keine so hohen Fluggeschwindigkeiten erreichten wie im Freiland, wäre diese : Man muß berücksichtigen, daß das Flugziel auch optisch kontrolliert wird. Die Biene weiß also trotz des Windes, daß sie nicht $100 \mathrm{~m}$ sondern nur so und so viele $\mathrm{cm}$ zum Ziel fliegen muß. Sie befindet sich in derselben Situation wie eine Biene im Freien, die bald landen wird. Dabei wird sie schon von verhältnismässig schwachen Windböen - die sie im vollen Flug ohne weiteres überwindet - abgetrieben. Bei häufigen, starken Böen fliegt dieselbe Biene manchmal ihr Flugloch mehrmals vergeblich an. Sie hat ganz offensichtlich auf Grund der optischen Signale auf “ Vorsicht » geschaltet. Dadurch entgeht sie der Gefahr, bei plötzlichem Aussetzen der Strömung gegen die Wand zu prallen. 
Die volle Fluggeschwindigkeit ist also nur zu erwarten, wenn diese (reflektorischen ?) Hemmungen aus dem optischen System fehlen. (Ruttner, F. pers. Mitt.)

Eingegangen im Juni 1970

Reçu pour publication en juin 1970

\section{RÉSUMÉ}

Pour les recherches portant sur la cinématique du vol des insectes ceux-ci sont attachés par le thorax ou l'abdomen à une balance aérodymanique devant une soufflerie. Pour éviter ces conditions de vol peu naturelles on a essayé de dresser des insectes susceptibles de dressage - des abeilles - à effectuer des vols libres dans un canal d'aspiration. Les expériences ont été faites dans une chambre pour abeilles $\left(6 \mathrm{~m}^{2}, 25^{\circ} \mathrm{C}\right.$, température constante, humidité relative $50 \%$, éclairage fluorescent donnant 14 heures de jour avec phase crépusculaire de 20 minutes). La nourriture consistait en solution de sucre 2-molaire. La soufflerie utilisée (fig. 1) permettait d'atteindre une vitesse maximum du vent de $6 \mathrm{~m}$ par seconde dans la veine de $80 \times 12 \times 12 \mathrm{~cm}$. Les abeilles furent dressées à voler contre le vent, à atterrir sur la face interne du dernier des filtres de turbulence, à ramper vers le haut jusqu'à un orifice de sortie pratiqué latéralement dans la tuyère et, de là, à voler vers une coupelle de nourrissement placée à un mètre devant la tuyère et fortement éclairée. A partir de la coupelle, les abeilles rentraient à la ruche en volant à travers la chambre en utilisant une entrée latérale distincte de la sortie et munie d'un système automatique de sens unique.

Lorsque la vitesse de vol de l'abeille est égale et de sens opposé à la vitesse du vent, l'abeille est en vol stationnaire dans le canal et on peut l'observer et la filmer d'en haut, d'en bas ou par le côté. Le moment critique est celui du départ de la plateforme d'envol; seulement environ $2 \%$ des insectes s'envolent correctement et se maintiennent jusqu'à 20 secondes en vol libre dans le canal. Elles y volent avec une vitesse de 0 à $5,5 \mathrm{~m} / \mathrm{s}(0-20 \mathrm{~km} / \mathrm{H})$ avec une tendance prononcée à effectuer des mouvements de balancement latéraux. A l'air libre les vitesses sont plus élevées. Des marques éclairées, en forme de rayures alignées dans le sens du vol, et placées sous la veine vitrée n'ont eu jusqu'ici aucune influence sur le comportement de vol.

Les abeilles en vol libre ont été filmées par le côté. On a pu montrer que l'angle d'incidence du corps diminue lorsque la vitesse augmente (résultats statistiquement confirmés; test $\mathrm{t}$ ). La dispersion des valeurs de l'angle d'incidence du corps diminue lorsque la vitesse augmente (résultats statistiquement confirmés; test F) (tableau 1). De toute évidence l'angle d'indicence du corps est, pour des raisons d'ordre énergétique, d'autant plus exactement maintenu que la vitesse est plus grande.

\section{LITERATUR}

Heran H., 1959. Wahrnehmung und Regelung der Flugeigengeschwindigkeit bei Apis mellifica. Z. vergl. Physiol., 42, 103-163.

Heran H., Lindauer M., 1963. Windkompensation und Seitenwindkorrektur der Biene beim Flug über Wasser. Z, vergl. Physiol, 47, 39-55.

Herbst H. G., Freund K., 1962. Kinematik der Flügel bei ventilierenden Honigbienen. Deutsch. entomol. $Z$., N. F., 9, (I/II), 1-29.

Herolv E., 1960. Biene und Bienenzucht. Ehrenwirth, München. 
Hollick F. S. J., 1940. The flight of the dipterous fly Muscina stabulans Fallén. Phil. Trans. B., $230,357-390$.

Jensen M., 1956. Biology and physics of locust flight. III. The aerodynamics of locust flight. Phil. Trans, B., 239, 511-552.

NachtigalL W., 1966. Die Kinematik der Schlagflügelbewegungen von Dipteren. Methodische und analytische Grundlagen zur Biophysik des Insektenflugs. Z. vergl. Physiol., $52,155-211$.

Neuhaus W. Wohlgemuth R., 1960. Über das Fächeln der Bienen und dessen Verhältnis zum Fliegen. Z. vergl. Physiol., 43, 615-641.

Pringle J. W. S., 1961. The function of the direct muscles in the bee. Proc. XI. Int. Congr. Entomol. Vienna $1960,1,660$.

RenNer M., 1955. Uber die Haltung von Bienen in geschlossenen künstlich beleuchteten Räumen. Naturwissenschaften, 42, 539-540.

Renner M., 1957. Neue Versuche über den Zeitsinn der Honigbienne. Z. vergl. Physiol., 40, 85-118.

Voged S., 1966. Flight in Drosophila. I. Flight performance of tethered flies. J. exp. Biol., 44, 567-578.

Weber E., 1964. Statistisches Rechnen für Biologen. 5. Aufl. Fischer-Leipzig.

Widmann R., Staatsexamensarbeit, Nat. Fak. München, unpubliziert.

Wohlgemuth R., 1962. Die Schlagform des Bienenflügels beim Sterzeln im Vergleich zur Bewegungsweise beim Fliegen und Fächeln. Z, vergl. Physiol., 45, 581-589 (1962).

ZarNack W., 1970. Kinematik der Flügelschlagbewegung bei Locusta migratoria (L.), $Z$ vergl. Physiol., (im Druck; Arbeitstitel).

Anschriften der Verfasser :

Prof. Dr. W. Nachtigali, Zoologisches Institut der Universität des Saarlandes, 66 Saarbrücken.

R. WidmanN, 8 München 82, Lohnrösselerweg 15.

Prof. Dr. M. RenNer, Zoologisches Institut der Universität München, 8 München 2, Luisenstrasse 14. 